\title{
NECESIDADES PEDAGÓGICAS PARA LA ENSEÑANZA EN ENTORNOS VIRTUALES DE ENSEÑANZA APRENDIZAJE
}

\author{
SÃO LUÍS/MA ABRIL/2018
}

\author{
Maira Rejane Oliveira Pereira \\ - UEMA - mairajane@gmail.com \\ Eliza Flora Muniz Araujo \\ - UEMA - eliza.uemanet@gmail.com \\ Ilka Marcia R. de Souza Serra - UEMA - ilka.tt@gmail.com \\ Jorge Alberto Alárcon Leiva - UTALCA - j.alarconleiva@gmail.com
}

Tipo: Investigação Científica (IC)

Natureza: Relatório Final de Pesquisa

Categoria: Pesquisa e Avaliação

Setor Educacional: EDUCAÇÃO SUPERIOR

\begin{abstract}
RESUMO
Este estudio se realizó en el Núcleo de Tecnología para Educación de la Universidad Estadual del Maranhão con el objetivo de diagnosticar las necesidades pedagógicas de los tutores que actúan en los cursos a distancia de esa institución. La investigación se basa en el método misto como alternativa más adecuada la comprensión de los fenómenos estudiados, con la combinación de técnicas de naturaleza cuantitativa y cualitativa que permite un análisis más completo del objeto de estudio. Para la recogida de los datos se utilizó: investigación documental, cuestionarios, entrevistas semiestructuradas y observación del Aula Virtual. Los resultados del estudio señalan que los tutores requieren de una formación continua que les permitan desarrollar las competencias necesarias a la enseñanza en entornos virtuales. Un programa de formación en ese contexto debe estar ajustado a las necesidades profesionales de los tutores y de la institución.
\end{abstract}

Palavras-chave: Tutoría. Necesidades. Educación a distancia. Formación. 


\section{Introducción}

Los cursos en la modalidad EaD tiene desarrollado mejoría en los procesos, en las herramientas y metodologías empleadas. Son cursos con características peculiares que por medio de las nuevas tecnologías ofrecen a profesores y alumnos, un conjunto de herramientas que dinamizan y facilitan el proceso de aprendizaje.

En ese contexto es urgente la necesidad de elaborar programas de formación que fomente el desarrollo de competencias propias de la docencia para el contexto virtual. Para ello, se requiere conocer y analizar las necesidades formativas declaradas por los propios docentes, corresponsabilizándoles en el proceso, lo que favorecerá el logro del desarrollo profesional y la satisfacción de sus reales necesidades de formación.

La motivación del estudio nació de la experiencia vivida por los investigadores como participantes del equipo multidisciplinar en esa institución. Experiencia que estuvo marcada por la dificultad en consolidar resultados significativos y duraderos, reflejados en el desempeño de los profesionales que ahí actúan. Sumado a eso, hay que destacar la relevancia permanente de la temática, formación de profesores, para el logro de una educación de calidad alineada con las demandas de la sociedad contemporánea.

El presente trabajo evidencia la importancia del desarrollo de investigaciones en el campo de la EaD y tiene com objetivo el análisis de las necesidades formativas de los profesionales que actúan en la enseñanza a distancia, como forma de elevar la calidad de la educación ofertada.

\section{Marco Teórico}

El proceso de aprendizaje puede ser entendido como el resultado de la interacción entre contenido, estudiante y profesor, sin embargo, en el contexto de la educación a distancia en que el proceso de aprendizaje es mediado por la tecnología, algunos autores apuntan otras versiones de ese triángulo educacional. Lombard (2003) propone uno tetraedro de integración cuyos polos son el estudiante, el maestro, el conocimiento y el cuarto polo, los dispositivos tecnológicos, a que él llama de "dispositifcyber-prof". Entonces, para apoyar la auténtica aprendizaje en la educación virtual se hace imperativo proporcionar los soportes adecuados.

En la Educación a Distancia, el énfasis está en la interactividad que ocurre entre estos distintos elementos y en el papel que cada uno desarrolla. Predomina un abordaje constructivista, aquí entendido como aquel que el conocimiento no es dado, en ninguna 
instancia, como algo acabado, se constituye como resultado de la interacción del individuo con el medio físico y social (BECKER, 1993); en que el estudiante debe asumir el control por su aprendizaje, creando o adueñándose de mecanismos existentes en el ambiente virtual (dispositivos tecnológicos) para generar su propio conocimiento. En ese escenario el estudiante adquiere un papel mucho más activo, mientras el profesor es un facilitador del proceso aprendizaje enseñanza, un mediador del conocimiento, que les orienta en el entramado de informaciones presentes en la red.

Esa complejidad es especialmente contundente en lo que dice respecto a los docentes que ahí actúan. El profesor en ese tipo de enseñanza asume distintos papeles dependiendo de las tareas que desarrolla: docente formador, docente autor, docente investigador, docente tutor, y otros. Se puede inferir aun que el papel del docente en el entorno virtual de aprendizaje excede la simple orientación pedagógica o cognitiva de los estudiantes. Hay una especial porción afectiva en su rol, una vez que es éste quien mantiene el contacto constante con el estudiante, siendo responsable de crear un ambiente afectivo capaz de superar el aspecto frio de la virtualidad. El docente virtual debe establecer clima cordial, humano, provocador (problematizador) que contribuya al proceso de aprendizaje (EMERENCIANO, SOUSA y FREITAS, 2001).

Así que, el buen desempeño de la función del docente en EVEA requiere más que conocimiento conceptual, se exige de este identificar los distintos ritmos de aprendizaje de cada estudiante, apropiarse de la TDIC, dominio de técnicas e instrumentos de evaluación, tener habilidades de investigación, utilizar nuevos esquemas mentales para crear una nueva cultura indagadora y plena en procedimientos de creatividad y tener disponibilidad para intervenir en cualquier momento (BENTES, 2009).

Ese contexto dinámico donde actúa el docente virtual crea constantemente nuevas necesidades, lo que exige del profesor una permanente relectura de su papel frente a toda esa vicisitud. Partiendo de un proceso de reflexión crítica, debe despertar para la necesidad de mejorar su praxis pedagógica con el fin de atender las exigencias de estos escenarios cambiantes. En ese proceso el análisis de necesidades es elemento primordial para el conocimiento de la realidad actual y del nivel deseable que se persigue.

La concepción de necesidades se tomó de diferentes autores: Kauffman y English (1979), Stufflebeam (1985), Bradshaw (1972), Monette (1977), Boone, Safrit y Jones, (2002), Beatty (1981), Pérez-Campanero (1994), entre otros.

Kauffman y English (1979) define necesidad como la discrepancia entre los resultados 
actuales y los resultados deseados. La evaluación que se hace de las necesidades es un proceso formal que pone en orden de prioridad las brechas identificadas.

Para Stufflebeam, McCormick, Brinkerhoffy Nelson (1985) cualquier evaluación de necesidades debe ser interpretada dentro de un contexto específico, este autor presenta cuatro visiones de necesidades: discrepancia, democrática, analítica, diagnóstica.

Además del abordaje de necesidad como discrepancia, carencia o deficiencia comúnmente aplicado, el término también es empleado como problema. La perspectiva de Pérez-Campanero, define necesidad como el resultado inadecuado que se debe intervenir para corregir la situación. Para la autora el "análisis de necesidades es un estudio sistemático de un problema, que se realiza incorporando información y opiniones de diversas fuentes, para tomar decisiones sobre lo que hay que hacer a continuación" (PÉREZ-CAMPANERO, 1994, p. 25).

Emerge de esas definiciones la noción de que necesidad es el efecto acumulativo de una serie de factores psicológicos, sociales, culturales, profesionales, etc. que no tiene un sentido absoluto una vez que son resultantes de juicio de valor, del tiempo considerado y del contexto en que se está analizando (ARÀNEGA, 2013). Es un concepto complexo, multidimensional y dinámico que pode ser analizado desde distintas perspectiva.

El abordaje que sobresale en el presente trabajo pode ser resumida como discrepancias - con destaque para la perspectiva del otro (en ese caso la institución), que define cual es el estado que se desea alcanzar (visión prescriptiva); como problema - que puede estar más bien direccionada a la visión del individuo (sentida) y emerge de la práctica diaria.

En el contexto de la educación, el análisis de necesidades es relevante pues ofrece un abordaje racional para la determinación de prioridades y asignación de recursos (STUFFLEBEAM et al., 1985).

Para Pérez-Campanero (1994) el análisis de necesidades es un estudio sistemático que utiliza datos representativos de la realidad y de las personas implicadas, siendo un proceso importante para generar datos en que fundamentar la toma de decisión. Como plantea Kauffman y English (1979) el uso de los instrumentos y técnicas adecuados para detectar las necesidades puede ser determinante para el suceso del proyecto a que se propone. Siendo elemento primordial para el conocimiento de la realidad actual y del nivel deseable que se persigue. 


\section{Metodologia}

Debido a los múltiples enfoques metodológicos posibles en la esfera del análisis de necesidades, se optó por el método misto en la investigación como alternativa más oportuna para la comprensión de los fenómenos estudiados, con la conjugación de técnicas de naturaleza cuantitativa y cualitativa que permite un análisis más completo del objeto de estudio (CRESWELL y PLANO CLARK, 2011).

El abordaje cualitativo orientó al análisis de la realidad de los actores del contexto educativo en cuestión (CRESWELL, 2012), las interacciones, problemáticas y fortalezas presentes en la práctica pedagógica de los tutores virtuales, tanto como la elaboración y validación de los instrumentos de recolección de datos. El carácter cualitativo opera en un universo de significados, motivos, aspiraciones, creencias, valores y actitudes, lo que corresponde a un espacio más profundo de las relaciones, procesos y fenómenos (MINAYO, 2001).

Y el cuantitativo se refiere especialmente a análisis de los datos referentes a la aplicación del cuestionario y el establecimiento de frecuencia de conductas lo que generó los insumos que orientaran las entrevistas y observaciones realizadas posteriormente.

Del punto de vista de los objetivos, este estudio se caracteriza por ser del tipo descriptivo pues expone las características del profesor-tutor que actúa en entornos virtuales de aprendizaje, identificando las necesidades formativas de ese profesional.

Recogida y análisis de los datos

La población del estudio está constituida por tutores que desempeñan su función en carreras en la modalidad a distancia en el Núcleo de Tecnologías para la Educación de la Universidad Estadual de Maranhão, y por las coordinadoras de tutoría de esta institución. Para la recogida de los datos los seguientes instrumentos y técnicas: cuestionarios a los tutores con el objetivo de identificar la percepción de sus necesidades pedagógicas para el entorno virtual; entrevista semi-estructura con las coordinadoras de tutoría procurando percibir como estas evalúan la actuación de los tutores, resaltando debilidades y fortalezas del equipo; observación del aula virtual, que posibilitó analizar la vivencia de los tutores directamente en el EVEA. Para el análisis de los datos se utilizó técnicas cualitativa y cuantitativa, com apoyo del software SPSS, para el análisis descriptivo, y Atlas TI versión 7.5, para el análisis de contenido. 
4 Resultados y discusiones

Las distintas fuentes de informaciones e instrumentos utilizados en la investigación y la posterior triangulación de los resultados posibilitaron diagnosticar las necesidades pedagógicas de los docentes para la enseñanza en EVEA.

La dimensión pedagógica engloba variados e importantes aspectos del hacer docente que ganan complejidad cuando se considera el contexto virtual en que actúan. Entre esas acciones se puede destacar especialmente, el apoyo al proceso de aprendizaje, orientación en las situaciones de aprendizajes, retroalimentación evaluativa, etc., todo desde una mirada de las tecnologías digitales de información y comunicación y la capacidad de traducir su potencialidad en resultados prácticos conforme dado modelo educativo. Según Behar (2013) el modelo pedagógico en cual está enfocada la EaD corresponden a un sistema de premisas teóricas que representan, explica y orienta el currículo, las prácticas pedagógicas y las interacciones profesor/estudiante/objeto de estudio.

Para facilitar la exposición de los resultados, estos fueron divididos en tres subcategorías: mediación pedagógica, feedback, evaluación y seguimiento. A continuación se exponen los hallazgos de cada una de ellas.

\section{a) Mediación Pedagógica}

La mediación pedagógica es un factor imprescindible para el alcance de un aprendizaje significativo en un curso en la modalidad a distancia. Dentro del EVEA, los foros se traducen en el principal espacio de interacción y donde es posible analizar la calidad de la mediación.

En ese espacio fue posible observar que el docente presenta preocupación en dejar contribuciones teóricas, y muchas veces dando respuestas directas a las actividades planteada, sin conducir al estudiante a construir su conocimiento, esta característica de mediación afecta la autonomía del estudiante en elaborar sus inferencias, (des)construir sus ideas, profundizar el tema, o simplemente disminuye las posibilidades de su participación.

El resultado apunta que los tutores aun no dominan acciones relacionadas a la orientación y apoyo a los estudiantes. Aspectos relacionados a vincular los contenidos a las experiencias de los estudiantes, adoptar estrategias propias de la $\mathrm{EaD}$, fornecer recomendaciones metodológicas, adicionar informaciones complementarias, y apoyar al 
estudiante en la organización de su estudio aun no son frecuentes o sistemáticas en la práctica de los docentes.

En cuanto a promover la autorreflexión y autonomía de los estudiantes, se identificó que la mediación llevada a cabo tiene fuerte connotación en la transmisión de contenidos, y no predomina un lenguaje dialógica. La mayoría de las interlocuciones observadas en el aula virtual no fue pautada en cuestionamientos, o retos que llevase al estudiante a la búsqueda de nuevas informaciones, oponerse a afirmaciones hechas por los compañeros, etc. Los cuestionamientos estaban enfocados en el 'Qué', es decir, preguntas que no fomentan la interacción o reflexión.

Salmon (2002, apud MAURI y ONRUBIA, 2010) destaca como actuaciones del tutor, facilitar el proceso de construcción del conocimiento por medio del establecimiento de desafíos reales y posibles de ser abordados, promover y desarrollar la reflexión y maximizar el papel del aprendizaje on-line para cada participante y para la experiencia de aprendizaje en grupo. El docente como mediador y facilitador en el EVEA debe proporcionar espacios propicios al progreso formativo del estudiante en que éste pueda tornarse protagonista de su aprendizaje.

\section{b) Feedback}

Shute (2008, apud ABREU-E-LIMA y ALVES, 2011) asume feedback formativo en el contexto EaD como la revisión de información comunicada para el aprendiz con la intención de modificar su comportamiento o su modo de pensar con el objetivo de mejorar su aprendizaje, para eso necesita tener algunas características que incluyen factores como el tiempo, cantidad de información y, sobre todo, el lenguaje utilizado.

La presencia del docente en el entorno virtual es constante, segundo observado por las coordinadores este aspecto hay mejorado bastante asociado principalmente por el intensa acompañamiento que les hacen.

Fueron encontrados tres tipos principales de feedback: con respuestas monosilábicas o respuestas automáticas (Figura 13) que se repiten a todos los estudiantes evidenciando que el docente no discrimina las participaciones de los mismos, o dar respuestas breves solamente con el objetivo de mostrar su presencia en el foro ya que esta es una exigencia de la coordinación de tutoría; centrado en el contenido, presenta citas textuales, clarifica conceptos y profundiza la temática; y, en menor frecuencia, el feedback con sugerencias de mejoras, y con preguntas que conducen el estudiantes a la búsqueda de mayores informaciones, y reflexionar para comprender el tema. No fue 
registrado feedback negativo.

Con respeto a la frecuencia y constancia, se observó un papel activo de los tutores en dar a los estudiantes retroalimentación en tiempo oportuno. En la literatura no hay un consenso cuanto al tiempo ideal para emitir un feedback, según Abreu e Lima y Alves (2011) feedback constante y seguidos puede al final inhibir la participación de los estudiantes o llevarlos a respuestas apresuradas, a su vez la ausencia o escasez de feedback pueden llevar a un desánimo de los estudiantes al pensar que no están siendo acompañados. Así que debe ser considerado cada contexto, cada clase de estudiantes, la asignatura, etc., para decidir cual el ritmo que más adecuado.

c) Evaluación y seguimiento

Con respecto a la evaluación la triangulación de los resultados identificó que hay una preocupación de los docentes en ofrecer retroalimentación buscando la mejora de las actividades, sin embargo esta acción por veces es perjudicada por el hecho de muchos estudiantes enviaren las tareas al final del plazo establecido no siendo posible corregir los errores señalizados.

Los docentes presentan o han presentado en alguna ocasión fuerte intención a la obtención de la calificación como objetivo primero de las actividades, lo que es indicado como principal motivo para los estudiantes atendieren las solicitudes del mediador.

Aún con respeto a evaluación de las tareas, se identificó la falta de parámetros en la definición de la nota. Con clases enteras con notas 10 y otras con 7 o debajo de eso, sin que haya encontrado aspectos que justifiquen la alta diferenciación, evidenciando ausencia de planificaciones en la evaluación de resultados.

Vale destacar que en las carreras observadas hay una prevalencia de heteroevaluaciones, no estando presente en ninguna de las asignaturas analizadas la práctica de autoevaluación o coevaluación, hecho que está relacionado a las directrices definidas por las coordinaciones de las carreras y no al trabajo desarrollado por el docente.

En los foros el seguimiento se caracteriza por indicaciones individualizadas para profundizar los conocimientos, adecuar la escritura, seguir normas de la ABNT con respeto a citas, también se preocupan en orientar a los estudiantes en el uso del material de internet. 
5 Consideraciones finales

A partir de los análisis desarrollados acerca de las necesidades pedagógicas de los docentes en el entorno virtual de la Universidad Estadual del Maranhão fue posible componer las conclusiones que siguen.

Los tutores de la Universidad Estadual do Maranhão encuentran dificultad cuanto a la mediación de los estudiantes, no logrando concretizar el apoyo al proceso de aprendizaje, orientación del proceso formativo, profundizar y contextualizar el conocimiento. Expresan necesidad de desarrollar metodologías propias de la enseñanza a distancia para el seguimiento y evaluación de los aprendizajes, ampliar el significado de la evaluación, y aplicar estrategias que promuevan mayor autonomía, responsabilidad y pensamiento crítico en los estudiantes.

Mismo con el esfuerzo que el UEMAnet hace por medio de las formaciones, no hay un retorno satisfactorio para la mejoría de la calidad del trabajo del tutor. En ese sentido, se recomienda que se fortalezca los canales de comunicación con el equipo tutorial de modo a que los tutores sean coparticipantes del proceso y no solamente prestadores de servicios. Fortalecer la sistematización del proceso evaluativo de modo a optimizar el programa, estrategias y actividades y no solamente el registro de informaciones parciales. Y elaborar un programa formativo que conforme el perfil de competencias del tutor en línea con las necesidades formativas diagnosticadas en el equipo tutorial de la institución.

\section{REFERENCIAS}

ABREU-E-LIMA, D. M.; ALVES, M. N. O feedback e sua importância no processo de tutoria a distância. Pro-Posições [online], 2011, vol.22, n.2, pp.189-205.

ARÀNEGA, S. De la detección de las necesidades de formación pedagógica a la elaboración de un plan de formación en la universidad. ( $1^{a}$ ed.). Barcelona: Octaedro, $2013 . \quad$ Disponíble en: http://www.ub.edu/ice/sites/default/files/docs/qdu/25cuaderno.pdf

BECKER, F. O que é construtivismo. Ideias. São Paulo: FDE, n.20, p.87-93, 1993.

BENTES, R. F. A Avaliação do Tutor. In: LITTO, F.M.; FORMIGA, M. (Orgs.). Educação a Distancia: o estado da arte. São Paulo: Pearson Education do Brasil, 2009, p. 167-169. 
CRESWELL, J. W. Qualitative Inquiry and Research Design: Choosing Among Five Approaches. Thousand Oaks, CA: Sage, 2012.

CRESWELL, J. W.; PLANO CLARK, V. L. Designing and conducting mixed methods research. 2nd. Los Angeles: SAGE Publications, 2011.

MERENCIANO, M. S.; SOUSA, C. A. L.; FREITAS, L. G. Ser presença como educador, professor e tutor. Colabor@-REVISTA Digital do CVA - Ricesu, Brasília, v. 1, n. 1, p. 4-11, 2001.

Kaufman, R. A. y English, F.W. Needs Assessment Concept and Application. Englewood Cliffs: Educational Technology Publications. New Jersey: Educational Technology Publications Englewood, 1979.

LOMBARD, F. Du triangle de Houssaye au tétraèdre des TIC: comprendre les interactions entre les savoirs d'expérience et ceux de recherche. In: CHARLIER, B. et D. Peraya (dir.). Transformation des regards sur la recherche en technologie de l'éducation. Bruxelles: De Boeck, p. 137-154, 2007.

MAURI, T.; ONRUBIA, J. O professor em ambientes virtuais. In: COLL, C.; MONEREO, C. (Cols). Psicología da educação virtual: aprender e ensinar com as tecnologias da informação e da comunicação. (pp. 118-135). Porto Alegre: Artmed, 2010.

MINAYO, M. C. de S. (Org.). Pesquisa Social: Teoria, método e criatividade. 18 ed. Petrópolis: Vozes, 2001.

PÉREZ-CAMPANERO, M. P. Como detectar las necesidades de intervención socioeducativa. Madrid: Narcea, 1994.

STUFFLEBEAM, D. L., MCCORMICK, C. H., BRINKERHOFF, R. O., \& NELSON, C. O. Conducting educational needs assessment. Dordrecht. The Netherlands: KluwerNijhoff Publishing, 1985. 